\title{
Perfil dos portadores de tuberculose em um município de Minas Gerais
}

\author{
Profile of tuberculosis patients in a Minas Gerais municipality

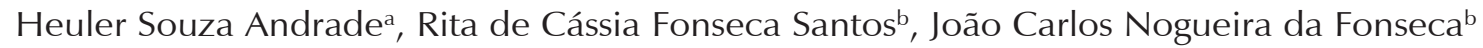 \\ a Enfermeiro. Mestre em Ciências pela Universidade Federal de São João del-Rei. Professor da Universidade do Estado de Minas Gerais (UEMG), Divinópolis (MG), Brasil. \\ b Enfermeiros graduados pela UEMG, Divinópolis (MG), Brasil.
}

RESUMO Introdução: A Tuberculose é uma doença de grande impacto social no mundo. Embora haja uma tendência de diminuição de seus índices no Brasil, existem fragilidades estruturais e na gestão dos serviços. As informações em saúde são consideradas essenciais para melhoria desse cenário.

Objetivo: Identificar o perfil do portador de tuberculose pulmonar no município de Divinópolis no período de 2010 a 2015.

Materiais e Métodos: Estudo descritivo, epidemiológico, quantitativo e de caráter histórico. A coleta de dados aconteceu através da consulta às fichas de notificação contidas no Sistema de Informação de Agravos de Notificação. A população elegível foram os casos novos de tuberculose pulmonar, dos residentes de Divinópolis, notificados entre os anos de 2010 a 2015.

Resultados: Foram notificados 104 casos novos de tuberculose pulmonar em residentes no município. A taxa de incidência variou de 6,04 a 10,06 casos por 100.000 habitantes. O maior número de notificações ocorreu no sexo masculino (67,3\%), em adultos acima de 50 anos $(57,7 \%)$, do ensino fundamental $(37,5 \%)$. Para a primeira baciloscopia de escarro 51,9\% dos pacientes tiveram resultados positivos. Quanto à cultura de escarro, a grande maioria $(70,2 \%)$ não realizou o exame. Como agravo associado à doença, o HIV positivo esteve presente em 9 notificações $(8,7 \%)$. Em relação à situação de encerramento a pesquisa apontou que 69 casos foram finalizados como cura e 8 abandonaram o tratamento.

Conclusão: Espera-se que o conhecimento do perfil dos casos possa auxiliar no planejamento de ações voltadas para o diagnóstico, tratamento e prevenção da doença.

Palavras-chave: tuberculose pulmonar; pacientes; saúde pública.

Introduction: Tuberculosis is a disease of great social impact in the world. Although there is a tendency to decrease its indices in Brazil, there are structural and management weaknesses in the services. Health information is considered essential to improve this scenario. Objective: To identify the profile of the pulmonary tuberculosis patients in the city of Divinópolis from 2010 to 2015.

Materials and Methods: Descriptive, epidemiological, quantitative and historical study. Data collection was performed through consultation of the notification sheets contained in the Notifiable Diseases Information System. The eligible population was new cases of pulmonary tuberculosis among residents of Divinópolis, notified between 2010 and 2015.

Results: 104 new cases of pulmonary tuberculosis were reported in residents of the municipality. The incidence rate ranged from 6.04 to 10.06 cases per 100,000 inhabitants. The highest number of reports occurred in males (67.3\%), adults over 50 years $(57.7 \%)$ and subjects with elementary school degree (37.5\%). For the first sputum smear, $51.9 \%$ of patients had positive results. As for sputum culture, the clear majority (70.2\%) did not take the exam. As an associated disease, HIV positive was present in 9 reports (8.7\%). Regarding the outcome situation, data indicated that 69 cases were completed as cured and 8 dropped the treatment out.

Conclusion: It is expected that the knowledge of the profile of the cases may help in planning actions aimed at the diagnosis, treatment and prevention of the disease.

Keywords: pulmonary tuberculosis; patients; public health. 


\section{INTRODUÇÃO}

A Tuberculose (TB) é uma das doenças mais antigas conhecidas pela humanidade, contudo ainda nos dias atuais possui um grande impacto social em todo planeta. De acordo com a Organização Mundial da Saúde (OMS), em 2015 a TB estava entre as dez maiores causas de morte em todo o mundo, classificando-se acima do HIV/AIDS. No mesmo ano, as estimativas foram que cerca de 10,4 milhões de pessoas foram acometidos pela doença e desses, 1,4 miIhões resultaram em óbito. Indivíduos que possuem baixas condições de vida e que não são imunocompetentes têm maiores chances de desenvolver a patologia ${ }^{1}$.

Dentre as ações globais relacionadas ao combate da TB pode-se citar o Tratamento Diretamente Supervisionado (DOTS), proposto pela OMS em 1993². No Brasil, em 1998, com a finalidade de ampliar o combate à TB, o Ministério da Saúde (MS) lançou o Plano Nacional de Controle da Tuberculose (PNCT), que se baseia na descentralização e horizontalização das ações de tratamento da doença, bem como de vigilância, prevenção e controle direcionadas para a atenção básica ${ }^{3}$.

Atualmente, o Brasil está entre os 22 países que concentram $80 \%$ dos casos de TB no mundo. Desse grupo, o país ocupa a $18^{a}$ posição em relação ao número absoluto de casos e a $22^{\text {a }}$ considerando o coeficiente de incidência. Entre os anos de 2005 a 2014 foram relatados no Brasil uma média anual de 75 mil novos casos de TB. De acordo com o Ministério da Saúde, em 2016 foram registrados 66.796 casos novos de TB no Brasil|, ${ }^{4}$.

O estado de Minas Gerais apresentou em 2016 uma taxa de incidência de 15,8/100 mil habitantes ${ }^{5}$. No município de Divinópolis-MG em 2012, a taxa de incidência da TB foi de 12,9 casos/100.000 habitantes, enquanto a taxa de mortalidade foi de 1,4 óbitos/100.000 habitantes e os índices de cura e abandono do tratamento foram de 57,1\% e 14,3\% respectivamente ${ }^{6}$.

Embora haja uma tendência de redução dos coeficientes de incidência e mortalidade por TB no Brasil ${ }^{5}$, estudos em diferentes regiões do país indicaram fragilidades estruturais e na gestão dos serviços como a baixa resolutividade da Atenção Primária à Saúde (APS) e deficiência nas ações de vigilância ${ }^{7-10}$. As informações em saúde são consideradas essenciais no que diz respeito ao planejamento de ações que garantam a qualidade da assistência e o controle da TB ${ }^{11}$.

Diante do cenário mundial e nacional da TB, conhecer o perfil dos pacientes diagnosticados com tuberculose no município de Divinópolis poderá auxiliar os atores que utilizam das informações em saúde na tomada de decisão para melhores direcionamentos das ações de promoção, prevenção e controle da doença. Desta forma, o presente estudo objetivou identificar o perfil dos casos novos de tuberculose pulmonar na cidade de Divinópolis no período de 2010 a 2015.

\section{MATERIAIS E MÉTODOS}

Trata-se de um estudo descritivo, epidemiológico, quantitativo, de caráter histórico realizado no município de Divinópolis - MG no ano de 2016. A cidade é a sede da Região Oeste de Saúde de Minas Gerais e é referência em saúde para 54 municípios. Sua população estimada em 2015 é de 230.848 habitantes. Segundo o Cadastro Nacional de Estabelecimentos de Saúde (CNES), a cidade possui 32 unidades de Estratégia de Saúde da Família (ESF), 10 Unidades de Saúde Tradicional (UST), 02 hospitais especializados e 01 Unidade de Pronto atendimento (UPA) $)^{4,12}$.

A coleta de dados aconteceu na Superintendência Regional de Saúde (SRS), através da consulta às fichas de notificação contidas no Sistema de Informação de Agravos de Notificação (SINAN). Foi realizada no mês de maio de 2016, pelos autores, após aprovação da diretoria da instituição. A população elegível para o estudo foram os casos novos de TB, na forma pulmonar, dos residentes de Divinópolis, notificados no SINAN entre os anos de 2010 a 2015. Considerou-se relevante estudar os últimos cinco anos para se ter um panorama da situação epidemiológica do município. Como caso novo considera-se o doente com tuberculose que nunca se submeteu à quimioterapia antituberculosa, fez uso de tuberculostáticos por menos de 30 dias, ou submeteu-se ao tratamento para tuberculose há cinco anos ou mais ${ }^{3}$.

Os dados extraídos do SINAN foram tabulados no software Microsoft Excel 2016. Considerou-se as características sócio-demográficas (sexo, etnia, idade e escolaridade, institucionalização e moradores de rua), laboratoriais (baciloscopia, radiografia de tórax e cultura de escarro), comorbidades (HIV) e a situação de encerramento. Para a análise descritiva dos dados foi realizada a distribuição de frequência das variáveis e calculado a taxa de incidência de tuberculose pulmonar bacilífera, a proporção de casos curados e proporção de casos que abandonaram o tratamento.

O projeto dessa pesquisa foi submetido ao Comitê de Ética em Pesquisa da Universidade do Estado de Minas Gerais e aprovado sob o parecer de número 1.796.296.

\section{RESULTADOS}

No período de 2010 a 2015 foram notificados 104 casos novos de tuberculose pulmonar em residentes do município de Divinópolis. A taxa de incidência desses últimos cinco 
anos variou de 6,04 a 10,06 casos por 100.000 habitantes

(Tabela 1).

O maior número de notificações da doença ocorreu no sexo masculino com $70(67,3 \%)$ casos. Em relação à faixa etária foi observado o predomínio em adultos acima de 50 anos totalizando $60(57,7 \%)$ casos. O ensino fundamental é o

Tabela 1. Incidência anual dos casos novos de tuberculose pulmonar em Divinópolis, no período de 2010-2015.

\begin{tabular}{|ccc|}
\hline Ano & $\mathbf{n}$ & $\begin{array}{c}\text { Taxa Incidência/ } \\
\mathbf{1 0 0 . 0 0 0} \text { hab. }\end{array}$ \\
\hline 2010 & 19 & 8,9 \\
\hline 2011 & 13 & 6,0 \\
\hline 2012 & 18 & 8,2 \\
\hline 2013 & 17 & 7,5 \\
\hline 2014 & 23 & 10,0 \\
\hline 2015 & 14 & 6,0 \\
\hline Total & 104 & \\
\hline
\end{tabular}

Fonte: SINAN.

Tabela 2. Características sócias demográficas dos casos novos de tuberculose em Divinópolis no período de 2010-2015.

\begin{tabular}{|c|c|c|}
\hline Características & $\mathbf{n}$ & $\%$ \\
\hline \multicolumn{3}{|l|}{ Sexo } \\
\hline Masculino & 70 & 67,3 \\
\hline Feminino & 34 & 32,7 \\
\hline \multicolumn{3}{|l|}{ Idade (anos) } \\
\hline 0 a 19 & 0 & 0 \\
\hline 20 a 29 & 9 & 8,7 \\
\hline 30 a 39 & 10 & 9,6 \\
\hline 40 a 49 & 24 & 23,1 \\
\hline 50 a 59 & 32 & 30,8 \\
\hline 60 e mais & 28 & 26,9 \\
\hline Ignorado & 1 & 0,9 \\
\hline \multicolumn{3}{|l|}{ Escolaridade } \\
\hline Analfabeto & 5 & 4,8 \\
\hline Ensino Fundamental & 39 & 37,5 \\
\hline Ensino Médio & 15 & 14,4 \\
\hline Ensino Superior & 2 & 1,9 \\
\hline Ignorado & 43 & 41,3 \\
\hline \multicolumn{3}{|l|}{ Raça } \\
\hline Branca & 45 & 43,3 \\
\hline Não Branca & 37 & 35,5 \\
\hline Ignorado & 22 & 21,2 \\
\hline \multicolumn{3}{|l|}{ Institucionalizados } \\
\hline Sim & 2 & 1,9 \\
\hline Não & 16 & 15,4 \\
\hline Ignorado/vazio & 86 & 82,7 \\
\hline \multicolumn{3}{|l|}{ Moradores de Rua } \\
\hline Sim & 0 & 0 \\
\hline Não & 17 & 16,3 \\
\hline Ignorado & 87 & 83,7 \\
\hline
\end{tabular}

Fonte: SINAN nível de escolaridade com maior ocorrência representando 39 $(37,5 \%)$ casos. Em relação à etnia a maioria se declara branco com 45 (43,3\%) notificações. Os resultados demonstraram que $2(1,9 \%)$ pessoas eram institucionalizadas e nenhuma estava em situação de rua. Contudo em mais de $80 \%$ das notificações o preenchimento dos campos referentes a esses dados foi ignorado (Tabela 2).

No que se refere à parte de diagnósticos, na primeira baciloscopia de escarro 54 (51,9\%) dos pacientes tiveram resultados positivos e 26 (25\%) foram negativos. Na segunda baciloscopia, 33 (31,7\%) apresentaram resultados positivos e 19 (18,3\%) negativos. Em 98 (94,2\%) casos o exame de Radiografia do tórax apresentou-se como suspeito. No que diz respeito à cultura de escarro, a grande maioria não realizou o exame, representado 73 (70,2\%) casos. Como agravo associado a TB, o HIV positivo está presente em 9 notificações (8,7\%) (Tabela 3 ).

Em relação à situação de encerramento a pesquisa apontou que 69 (66,3\%) casos foram finalizados como cura e 8 pacientes $(7,7 \%)$ abandonaram o tratamento (Tabela 4).

Tabela 3. Resultados de exames dos casos novos diagnosticados como tuberculose pulmonar no município de Divinópolis, 2010-2015.

\begin{tabular}{|c|c|c|}
\hline Variável & $\mathbf{n}$ & $\%$ \\
\hline \multicolumn{3}{|c|}{ Resultado da 1o Baciloscopia } \\
\hline Positivo & 54 & 51,9 \\
\hline Negativo & 26 & 25,0 \\
\hline Não realizada & 24 & 23,1 \\
\hline Não se aplica & 0 & 0,0 \\
\hline \multicolumn{3}{|c|}{ Resultado da $2^{\circ}$ Baciloscopia } \\
\hline Positivo & 33 & 31,7 \\
\hline Negativo & 19 & 18,3 \\
\hline Não realizada & 28 & 26,9 \\
\hline Vazio & 24 & 23,1 \\
\hline \multicolumn{3}{|c|}{ Resultado da Radiografia de Tórax } \\
\hline Suspeito & 98 & 94,2 \\
\hline Normal & 3 & 2,9 \\
\hline Outra Patologia & 1 & 1,0 \\
\hline Não realizado & 1 & 1,0 \\
\hline Vazio & 1 & 1,0 \\
\hline \multicolumn{3}{|c|}{ Resultado da Cultura } \\
\hline Positivo & 8 & 7,7 \\
\hline Negativo & 13 & 12,5 \\
\hline Em andamento & 10 & 9,6 \\
\hline Não realizada & 73 & 70,2 \\
\hline \multicolumn{3}{|c|}{ Resultado do Teste de HIV } \\
\hline Positivo & 9 & 8,7 \\
\hline Negativo & 75 & 72,1 \\
\hline Em andamento & 2 & 1,9 \\
\hline Não realizado & 18 & 17,3 \\
\hline
\end{tabular}

Fonte: SINAN 
Tabela 4. Situação de encerramento dos casos novos diagnosticados como tuberculose pulmonar no município de Divinópolis, 2010-2015.

\begin{tabular}{lcc}
\hline Variável & $\mathbf{n}$ & $\%$ \\
Cura & 69 & 66,3 \\
Abandono & 8 & 7,7 \\
Óbito por TB & 8 & 7,7 \\
Óbito por outras causas & 12 & 11,5 \\
Mudança de diagnóstico & 6 & 5,8 \\
Ignorado & 1 & 1 \\
\hline
\end{tabular}

\section{DISCUSSÃO}

Apesar das estratégias de controle empregadas pelo serviço de saúde, as taxas de incidência da doença no município apresentaram variações de ano para ano com acréscimos e declínios sequenciais. Tal oscilação pode estar relacionada a alguns fatores, dentre eles, a subnotificação dos casos de tuberculose e a fragilidade das ações de vigilância do programa municipal. Essas informações reforçam a importância de um trabalho consciente, com investimentos responsáveis e contínuos para redução satisfatória dos índices da TB no município ${ }^{13,14}$.

A predominância masculina em casos de TB não está bem esclarecida, porém alguns fatores podem estar relacionados com a baixa ou nenhuma procura aos serviços de saúde, maior exposição a determinados fatores de risco e menor importância ao autocuidado ${ }^{13,15}$. O fato de adultos acima de 50 anos concentrarem o maior número de casos pode estar relacionado à eficácia da vacinação BCG na infância e na maior suscetibilidade ao adoecimento da população nessa faixa etária ${ }^{16}$.

A baixa escolaridade na maioria das notificações analisadas pode influenciar negativamente na percepção e disseminação da doença ${ }^{17-19}$. Essa vulnerabilidade social dificulta o acesso à informação e a procura aos serviços de saúde. Além de restringir as condições favoráveis de vida e emprego, possibilita o aumento da pobreza e limita as boas condições de moradia e higiene ${ }^{20}$.

Em relação à etnia, na maioria das notificações os pacientes se intitularam brancos, em concordância com outros estudos. Essa informação pode estar associada ao modo como as pessoas se definem no ato do preenchimento da notificação, e não a uma predominância real da doença por raças ${ }^{19,21}$.

A situação de rua e a institucionalização podem ser considerados ambientes de grande vulnerabilidade devido à aglomeração de pessoas, pouca condição de higiene, nutrição precária, uso de álcool e drogas, e difícil acesso aos serviços de saúde. Esses fatores contribuem para a disseminação de doenças contagiosas como é o caso da TB, além de dificultar o tratamento. Embora tenha se identificado apenas 02 notificações de indivíduos institucionalizados e nenhuma em situação de rua, pode-se inferir que esse dado não reflete a situação do município em relação a essas variáveis devido ao grande número de notificações que não tiveram esse campo preenchido $^{22,23}$.

Em se tratando de diagnósticos de TB, a baciloscopia de escarro continua sendo o método prioritário que permite identificar cerca de $70 \%$ dos casos de TB Pulmonar. Contudo, identificou-se um número significativo de pacientes não realizaram a primeira e segunda baciloscopia de escarro. Considerando que esse é um exame de baixo custo, fácil acesso e com níveis consideráveis de assertividade para o diagnóstico da doença, seria importante que as equipes de saúde envolvidas no atendimento ao paciente portador ou suspeito de TB se atentassem para a realização desse exame a fim de melhorar esses índices ${ }^{24,25}$.

A cultura de escarro é considerada por alguns profissionais como um exame mais complexo, de maior custo e com maior tempo de realização. Apesar disso, é fundamental para a definição correta da forma bacteriana e identificação precoce de casos de resistência às drogas utilizadas. Estudos destacam a importância deste exame principalmente em casos de recidivas ou abandono, assim como casos de baciloscopia negativa ${ }^{24,26}$.

Embora a baciloscopia e a cultura de escarro sejam indicadas como ferramentas fundamentais para o diagnóstico e acompanhamento de TB pulmonar, nota-se que a radiografia de tórax é mais utilizada no diagnóstico diferencial e avaliação de tratamento da patologia ${ }^{27}$. O PNCT preconiza a utilização desse método para avaliação da doença e de lesões passadas, assim como para definição do tipo e extensão do comprometimento pulmonar ${ }^{3}$.

No que tange a comorbidade associada a TB, a infecção pelo Vírus da Imunodeficiência Humana (HIV) é considerada uma das mais graves. Embora a maioria dos casos notificados tenham feito o teste para HIV, é importante salientar que pessoas infectadas por HIV estão de 21 a 34 vezes mais propensas a desenvolver TB quando comparadas a população geral, sendo esta, a principal causa de morte por doença infecciosa em pessoas com HIV. Devido à resposta imunológica deficiente, pessoas que vivem com o vírus HIV, possuem maior risco de reativação da doença podendo aumentar o risco de transmissão do bacilo da TB para a população geral ${ }^{28,29}$.

Em relação a situação de encerramento, os índices encontrados não estão de acordo com o preconizado pela OMS1. Os achados do estudo mostraram que esses índices diminuíram significativamente entre os anos. Dentre 
os fatores mais apontados como causas para o abandono do tratamento, pode-se destacar os efeitos colaterais dos medicamentos utilizados, dificuldades no acesso aos serviços de saúde, pouco entendimento do processo saúde-doença, baixa escolaridade e contexto sociocultural. Também se pode associar esse fato ao início do desaparecimento dos sintomas, uma vez que devido a melhora clínica, o paciente se imagina curado e abandona o tratamento ${ }^{30-32}$.

Um fator limitante deste estudo foi o grande número de campos não preenchidos e ignorados nas fichas de notificação. Notificações não preenchidas ou preenchidas de maneira incompleta dificultam o conhecimento real da situação de saúde no país, prejudicando e impossibilitando ações de controle do governo por não saberem qual a extensão do problema ${ }^{33}$.

Pode-se identificar que a TB tem acometido na maioria dos casos, no município estudado, homens acima de 50 anos de idade, com pelo menos 6 anos de estudo e de raça branca. O conhecimento de algumas características, como os métodos diagnósticos e a proporção de cura e abandono do tratamento, dos casos novos de TB notificados no município sugere que pode haver problemas de planejamento e no processo de trabalho dos serviços. Em outros municípios brasileiros, estudos semelhantes identificaram que as atividades de planejamento conjunto, entre coordenação do programa e unidades básicas, aconteciam de forma esporádica9,10.

Desta forma, espera-se que, ao se tomar conhecimento dos resultados deste estudo, gestores e profissionais de saúde possam buscar melhores condições estruturais e de trabalho em prol de ações voltadas, especialmente, para o perfil identificado na tentativa alcançar os objetivos quanto à diminuição da magnitude da TB. Estudos avaliativos poderão apontar com mais precisão as deficiências relacionadas ao programa municipal de combate à doença.

\section{REFERÊNCIAS}

1. World Health Organization [homepage on the Internet]. Geneva: World Health Organization; [cited 2017 Jun 22]. Global tuberculosis report 2016. [Adobe Acrobat document, 293p.]. Available from: http://apps.who.int/iris/bitstream/10665/91355/1/978924156 4656_eng.pdf

2. Organização Pan-Americana Da Saúde. O apoio à implementação da Estratégia de Tratamento Diretamente Supervisionado (DOTS) para o combate à Tuberculose. Brasília: Organização Pan-Americana da Saúde; 2010.

3. Ministério da Saúde (BR). Secretaria de Vigilância em Saúde. Programa Nacional de Controle da Tuberculose. Manual de Recomendações para o Controle da Tuberculose no Brasil. Brasília: Ministério da Saúde; 2011.

4. Ministério da Saúde (BR). Departamento de Informática do SUS [Internet]. Brasília: Ministério da Saúde; 2014. Disponível em: http://www2.datasus.gov.br/DATASUS/index. php?area =01
5. Ministério da Saúde (BR). Secretaria de Vigilância em Saúde. Indicadores prioritários para o monitoramento do Plano Nacional pelo Fim da Tuberculose como Problema de Saúde Pública no Brasil. Bol Epidemiol (Brasília). 2017;8(8):1-11.

6. Silva MA. Tendência da tuberculose no município de Divinópolis - MG, de 2002 a 2012. [dissertação]. Divinópolis: Universidade Federal de São João Del Rei - UFSJ; 2014.

7. Assis EG, Beraldo AA, Monroe AA, Scatena LM, Cardozo-Gonzales RI, Palha PF, Protti ST, Villa TCS. A coordenação da assistência no controle da tuberculose. Rev Esc Enferm USP. 2012;46(1):111-8. https://doi.org/10.1590/S0080-62342012000100015

8. Feitoza DS, Clares JWB, Rodrigues LV, Almeida PC. Vigilância Epidemiológica no contexto do Programa de Controle da Tuberculose: limites e possibilidades. Rev Rene. 2012;13(5): 1066-74.

9. Heufemann NEC, Gonçalves MJF, Garnelo ML. Avaliação do programa de controle da tuberculose em cenário Amazônico: desafios em Coari. Acta Amaz. 2013;43(1):33-42. https://doi. org/10.1590/S0044-59672013000100005

10. Silva DM, Nogueira JA, Sá LD, Wysocki AD, Scatena LM, Villa TCS. Avaliação de desempenho de Serviços da Atenção Básica para o tratamento da tuberculose. Rev Esc Enferm USP. 2014;48(6):104453. https://doi.org/10.1590/S0080-623420140000700012

11. Guimarães EAA, Hartz ZMA, Filho AIL, Meira AJ, Luz AMP. Avaliação da implantação do Sistema de Informação sobre Nascidos Vivos em municípios de Minas Gerais, Brasil. Cad Saúde Pública. 2013;29(10):2105-18. https://doi.org/10.1590/0102$311 \times 00116312$

12. Ministério da Saúde (BR). Cadastro Nacional dos Estabelecimentos de Saúde do Brasil. Brasília: Ministério da Saúde; 2015.

13. Reis DC, Almeida TAC, Quites HFO, Sampaio MM. Perfil epidemiológico da tuberculose no Município de Belo Horizonte (MG), no período de 2002 a 2008. Rev Bras Epidemiol. 2013;16(3):592602. https://doi.org/10.1590/S1415-790X2013000300004

14. Almeida JB, Ponce MAZ, Wysocki AD, Santos MLSG, Vendramini SHF. A coordenação da assistência no controle da tuberculose na visão da equipe de enfermagem. Rev Enferm UFPE Online. 2016;10(Supl. 6):111-8.

15. Coutinho LASA, Oliveira DS, Souza GF, Fernandes Filho GMC, Saraiva MG. Perfil epidemiológico da tuberculose no Município de João Pessoa - PB, entre 2007 - 2010. Rev Bras Cienc Saúde. 2012;16(1):35-42. https://doi.org/10.4034/RBCS.2012.16.01.06

16. Caliari JS, Figueiredo RM de. Tuberculose: perfil de doentes, fluxo de atendimento e opinião de enfermeiros. Acta Paul Enferm. 2012;25(1):43-7. https://doi.org/10.1590/S0103-2100 2012000100008

17. Machado T, Fonseca AJ, Buenafuente SMF. Pleural tuberculosis in the state of Roraima, Brazil, between 2005 and 2013: quality of diagnosis. J Bras Pneumol. 2016;42(2):106-13. https://doi. org/10.1590/S1806-37562015000000082

18. Pinto ML, Silva TC, Gomes LC, Bertolozzi MR, Villavicencio LM, Azevedo KM, Figueiredo TM. Occurrence of tuberculosis cases in Crato, Ceará, from 2002 to 2011: a spatial analisys of specific Standards. Rev Bras Epidemiol. 2015;18(2):313-25. https://doi. org/10.1590/1980-5497201500020003 
19. Yamamura M, Santos-Neto M, Santos RAN, Garcia MCC, Nogueira JA, Arcêncio RA. Epidemiological characteristics of cases of death from tuberculosis and vulnerable territories. Rev. Latino-Am. Enfermagem. 2015 ;23(5):910-8. https://doi.org/10.1590/01041169.0450 .2631

20. Neto RJP, Gadelha RRM, Herzer TL, Peres DA, Leitão TMJS, Façanha MC, Holanda CN, Girão ES, Nogueira CMO, Alencar CH. Características clínico-epidemiológicas de pacientes com coinfecção HIV/tuberculose acompanhados nos serviços de referência para HIV/AIDS em Fortaleza, Ceará, entre 2004 e 2008. Cad Saúde Coletiva. 2012;20(2):244-9.

21. Ferraz AF, Valente JG. Epidemiological aspects of pulmonary tuberculosis in Mato Grosso do Sul, Brazil. Rev Bras Epidemiol. 2014;17(1):255-66. https://doi.org/10.1590/1415-790X2014 00010020ENC

22. Ministério da Saúde (BR). Secretaria de Atenção à Saúde. Departamento de Atenção Básica. Manual sobre o cuidado à saúde junto à população em situação de rua. Brasília: Ministério da Saúde; 2012.

23. Valença MS, Possuelo LG, Cezar-Vaz MR, Silva PEA. Tuberculose em presídios brasileiros: uma revisão integrativa da literatura. Ciênc Saúde Colet. 2016;1(7):2147-60. https://doi.org/10.1590/141381232015217.16172015

24. Augusto CJ, Carvalho WS, Goncalves AD, Ceccato MGB, Miranda SS. Características da tuberculose no estado de Minas Gerais entre 2002 e 2009. J Bras Pneumol. 2013;39(3):357-64. https://doi. org/10.1590/S1806-37132013000300013

25. Sicsú AN, Salem JI, Fujimoto LBM, Gonzales RIC, Cardoso MSL, Palha PF. Intervenção educativa para a coleta de escarro da tuberculose: um estudo quase experimental. Rev Latino-Am. Enfermagem. 2016; 24:e2703. https://doi.org/10.1590/1518-8345.0363.2703

26. Ministério da Saúde (BR). Secretaria de Atenção à Saúde. Departamento de Atenção Básica. Tuberculose na Atenção Primária à Saúde. Brasília: Ministério da Saúde; 2011.
27. Ozsahin SL, Arslan S, Epozturk K, Remziye E, Dogan OT. Radiografia torácica e bacteriologia na fase inicial de tratamento de 800 pacientes masculinos com tuberculose pulmonar. J Bras Pneumol. 2011 May-Jun;37(3):294-301. https://doi.org/10.1590/S180637132011000300004

28. Lemos LA, Fiuza MLT, Reis RK, FAC, Gir E, Galvão MTG. Adherence to antiretrovirals in people coinfected with the human immunodeficiency virus and tuberculosis. Rev. Latino-Am. Enfermagem. 2016;24:e2691. https://doi.org/10.1590/1518-8345. 0537.2691

29. Magnabosco GT, Lopes LM, Andrade RLP, Brunello MEF, Monroe AA, Villa TCS. Controle da Tuberculose em pessoas vivendo com HIV/AIDS. Rev Latino-Am Enfermagem. 2016;24:e2798. http:// dx.doi.org/10.1590/1518-8345.1187.2798

30. Couto DS de, Carvalho RN, Azevedo EB de, Moraes MN de, Pinheiro PGOD, Faustino EB. Fatores determinantes para o abandono do tratamento da tuberculose: representações dos usuários de um hospital público. Saúde Debate. 2014;38 (102):572-81. https://doi. org/10.5935/0103-1104.20140053

31. Silva EA, Anjos UU dos, Nogueira JA. Modelo preditivo ao abandono do tratamento da tuberculose. Saúde Debate. 2014;38(101):200-9. https://doi.org/10.5935/0103-1104.20140018

32. Silva PF, Moura GS, Caldas AJM. Fatores associados ao abandono do tratamento da tuberculose pulmonar no Maranhão, Brasil, no período de 2001 a 2010. Cad Saúde Pública. 2014;30(8):1745-54 https://doi.org/10.1590/0102-311X00124513

33. Pinheiro RS, Andrade VL, Oliveira GP. Subnotificação da tuberculose no Sistema de Informação de Agravos de Notificação (SINAN): abandono primário de bacilíferos e captação de casos em outras fontes de informação usando linkage probabilístico. Cad. Saúde Pública. 2012;28(8):1559-68. https://doi.org/10.1590/S0102$311 \times 2012000800014$ 